\title{
Assessing nocturnal sleep patterns in Latino mother-child dyads in a community setting - Lessons learned from a feasibility study
}

\author{
Martina R. Gallagher ${ }^{1}$, Karen Thomas ${ }^{2}$ \\ 1. University of Texas Health Science Center at Houston School of Nursing, Houston, TX, USA. 2. University of Washington \\ School of Nursing, Seattle, WA, USA.
}

Correspondence: Martina R. Gallagher. Address: UTHSC-Houston School of Nursing, 6901 Bertner Ave., Ste 740, Houston, Texas 77030, USA. Email: martina.r.gallagher@uth.tmc.edu

Received: January 29, 2013

Accepted: March 21, 2013

Online Published: April 16, 2013

DOI : $10.5430 /$ cns.v1n2p58

URL: http://dx.doi.org/10.5430/cns.v1n2p58

\section{Abstract}

Background: Latinos experience high rates of obesity and related sequelae such as diabetes and cardiovascular disease. Short sleep duration has been associated with obesity in children and adults, but most studies examining the association focused on non-Latinos. We do not know whether sleep assessment tools such as actigraphy and sleep diaries could be used with Latino mother-child dyads in community settings. Therefore, this feasibility study evaluated adherence to and acceptability of actigraphy and sleep diaries as tools for assessment sleep in Latino mothers and preschool children in a community setting.

Methods: Using a descriptive design, 10 low-income, Spanish-speaking mothers and their 2- to 5-year-old children were recruited from the community. Mother-child dyads wore actigraphy monitors and mothers completed sleep diary for 5 days. Adherence was evaluated by assessing the percentage of data missing from the actigraph and completed sleep diaries. Maternal acceptability was evaluated by a survey.

Results: There was minimal missing actigraphy data for a few children during the daytime, but not during the night time. Participants described some of the difficulties encountered in the study, including worries about the safekeeping of research equipment, the personal discipline needed to record data, and the confusion experienced using part of the sleep diaries.

Conclusion: Based on the results from this study, future sleep studies of Latina mothers and their children can be designed to increase adherence, improve acceptability, and minimize burden.

\section{Key words}

Latino, Sleep duration, Adherence, Actigraphy, Sleep diaries, Maternal, Child

\section{Introduction}

Latinos make up $16 \%$ of the U.S. total population ${ }^{[1]}$ and they experience high rates of overweight and obesity. These high rates are especially true for children ${ }^{[2]}$ and women of Mexican descent ${ }^{[3]}$. Obesity is the combined result of increased consumption of foods that are calorie dense and inadequate physical activity ${ }^{[4,5]}$. However, there is epidemiologic 
evidence that short sleep duration is also associated with an increased risk of weight gain in adults ${ }^{[6,7]}$ and children ${ }^{[7,8,9]}$.

Several laboratory and a few epidemiologic studies have attempted to tease out the reasons for the association between short sleep duration and obesity. From laboratory studies, there is evidence that short sleep duration results in decreased insulin sensitivity ${ }^{[10,11,12]}$, poor appetite regulation ${ }^{[13,14]}$ and increased food intake ${ }^{[14,15]}$. Individuals exposed to short sleep duration ( $<6$ hours per night) experienced an increased caloric intake via increased snacking in laboratory conditions ${ }^{[16]}$ and a large population study ${ }^{[17]}$. Although research in this area is slowly emerging, short sleep duration may also contribute to obesity by influencing levels of physical activity. Women who experience short sleep duration suffer from fatigue and sleepiness, which can result in decreased physical activity ${ }^{[18]}$. When individuals experience short sleep duration, there is an increase in the circulation of IL-6 and TNF-alpha ${ }^{[19,20]}$, which may result in feelings of fatigue and sleepiness. Schmid et al. ${ }^{[21]}$ found a relationship between short sleep duration and physical activity in healthy men, found that short sleep duration resulted in a decrease a daytime physical activity level. In a non-laboratory setting, young children who slept $<9$ hours per night were less active and had a higher BMI, than children who slept $>10$ hours per night ${ }^{[22]}$. Two limitations of laboratory and epidemiologic studies are that they focused on non-Latino populations and sleep duration measures were mostly based on self-report. Therefore, methodological issues that may influence sleep research in Latinos have only begun to emerge.

Latino children experience high rates of overweight and obesity ${ }^{[2]}$. In the Latino culture mothers shape their family's lifestyle habits such as diet and physical activity ${ }^{[23,24]}$, therefore future interventions to prevent obesity in children should incorporate healthy sleep education for Latino mothers. However, few studies have examined how mothers shape children's sleep patterns and behaviors in the home environment. To understand how to incorporate healthy sleep education as a way to address obesity in Latino children, it is important to study maternal and child sleep behaviors in a home and community setting. One challenge to studying sleep in Latinos is that it is not known if current methods of assessing sleep, such as with wrist actigraphy and sleep diaries, are culturally acceptable to this vulnerable population.

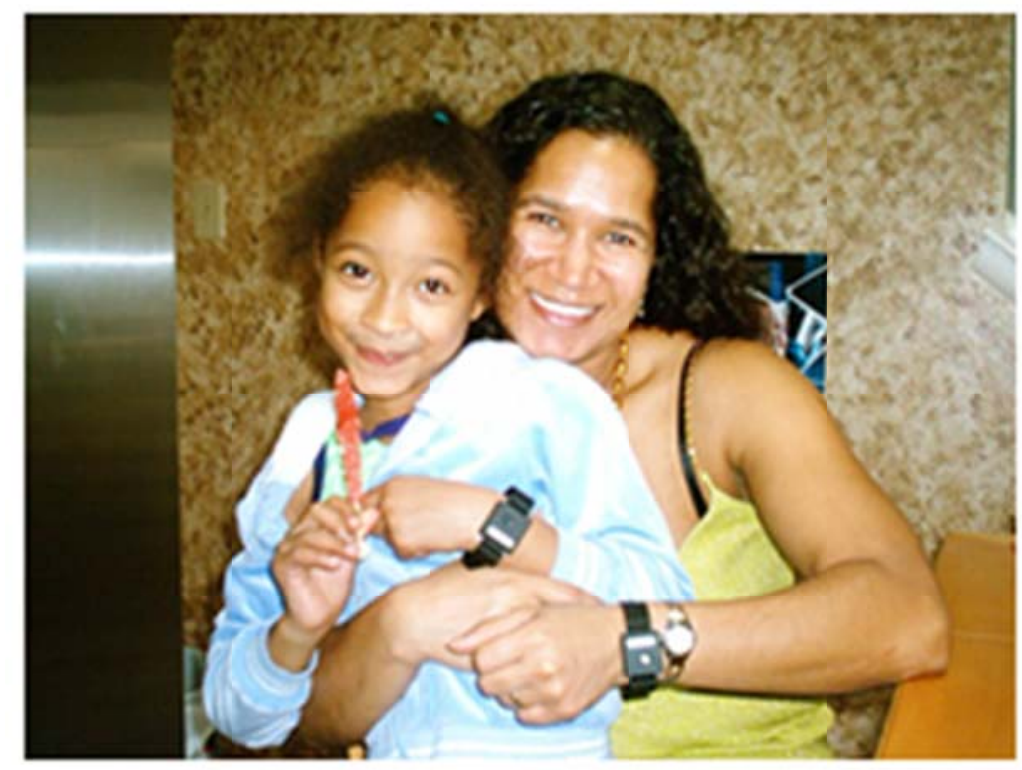

Figure 1. Mother and child dyad wearing wrist actigraph (Actigraphs courtesy of Actiwatch, Mini Mitter, Sun River, OR)

There are several articles that address methodological issues of using actigraphy ${ }^{[25,26,27]}$, yet these articles do not address the sociocultural factors that may prevent Latinos from participating in sleep research in a community setting. For 
example, during the early 2000's in the U.S., the Immigration and Naturalization Service (INS) began using electronic ankle bracelets to monitor undocumented immigrants who did not have criminal records ${ }^{[28]}$. Wearing an actigraph to record sleep (See Figure 1), although different from the electronic ankle monitors, could be viewed by community members as meaning that the participant is an undocumented immigrant or a criminal. This social stigma could result in decreased recruitment or following study protocols in sleep research studies that take place in Latino community settings.

Furthermore, few studies have examined sleep patterns concurrently in Latino mother-child dyads. Latino mothers have sociocultural considerations and family expectations that can influence the confidence needed to participate in a health research study ${ }^{[29]}$. It is not known if concurrently assessing sleep patterns in Latino mothers and children will increase the respondent burden that some participants experience in sleep studies that use actigraphy and sleep diaries ${ }^{[30]}$. Respondent burden can occur if participants perceive a psychological, physical, or financial hardship in relation to processes in a research project ${ }^{[31]}$. The purpose of this study evaluated adherence to and acceptability of actigraphy and sleep diaries in Latino mother-child dyads in a community setting, as a first step in establishing protocols for data collection on the relationship of sleep duration to obesity and other potential variables in Latino families. This knowledge will also will inform future recruitment and retention strategies for conducting biobehavioral research in a Latino community setting.

\section{Method}

\subsection{Design and subjects}

This feasibility study used a descriptive research design to evaluate adherence and maternal acceptability of actigraphy and sleep diaries with Latino mothers and their preschool children. The study was approved by the institutional review board of the sponsoring university and the community center where participants were recruited. Convenience sampling was used for this study. Women who self-identified as being of Latino descent, between the ages of 18-40, who had a child between the ages of 2 and 5 years, and who stated they could read and write in Spanish or English were eligible to participate in the study. Women who were pregnant; diagnosed with serious mental illness; taking sedative medications for sleep; working nights or rotating shifts; or had a child diagnosed with major genetic, medical, or psychological/behavioral problems were excluded from the study. Consent forms and all other subject materials were made available in Spanish and English.

\subsection{Recruitment and data collection protocol}

Participants were recruited from a community center that provided services such as English as a second language and parenting classes to Latino families in Seattle, WA. To make families aware of the study, flyers were posted in the community center. In addition, the primary author gave a brief presentation in various classes to introduce the study. Potential participants contacted the investigator by phone if they were interested in participating in the study. The participants were then screened, and if they met the inclusion criteria, a meeting was scheduled at a time and place that was convenient for the participant and her child. All of the participants preferred to have the data collection take place in their homes.

Data collection using actigraphy and the sleep diaries occurred over 5 week nights/days in the participants' homes. In order to control for unpredictable weekend sleep schedules data collection started on a Sunday evening and ended on a Friday morning. Once women participating in the study gave written consent and preschool children gave verbal assent, the following study protocol was followed (See Figure 2).

The demographic sheet was administered. Written and verbal instructions on how to use the Maternal/Child Sleeping Diary and the actigraphy monitor were provided to the mother. The mothers were instructed to wear the monitor continuously for 5 nights/days, except during bathing, and to use the sleep diary to record their and their child's sleep for 
the same time period. The mothers were also instructed to push the event marker on their and their child's actigraph at bedtime and wake time. Finally, the actigraph was placed on the mother and child's wrist of the non-dominant hand.

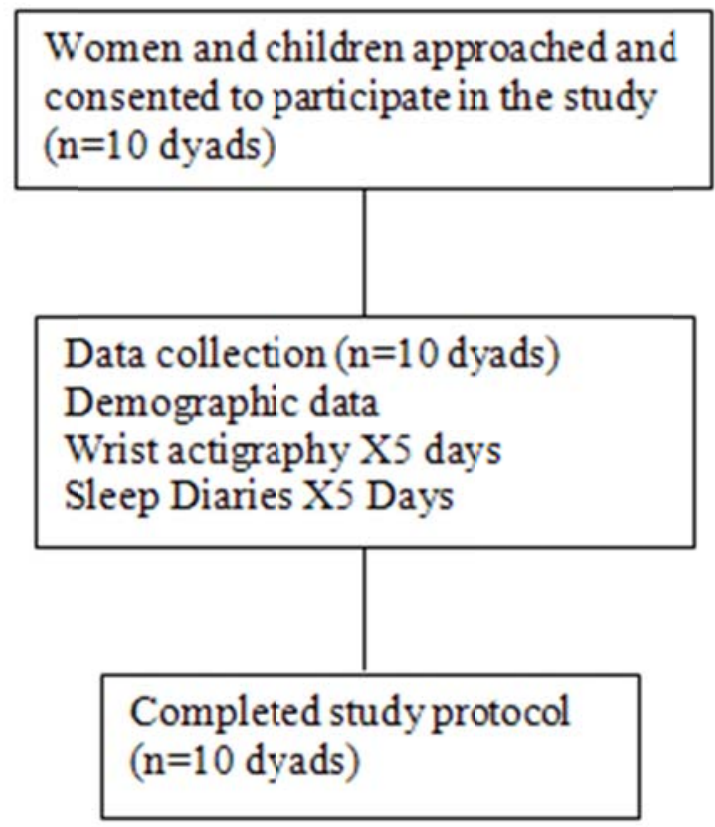

Figure 2. Flow chart of study recruitment and data collection procedures

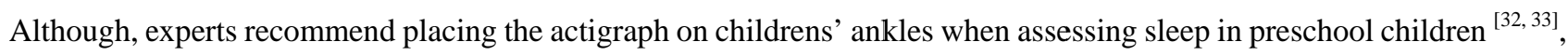
we found that the children in this study requested to have the actigraph placed on their wrist, just in the same manner as their mothers. To determine which hand was non-dominant, the investigator asked the mother with which hand did the child eat or draw on a regular basis. The participants were provided with a phone number to call if there were any questions or concerns regarding the Maternal/Child Sleep Diary or the actigraphy monitor. On the morning after the fifth night of data collection (Friday morning), the mother was instructed to remove her and her child's actigraph. The Maternal Acceptability Survey was administered and the actigraphs and sleep diaries were picked up after the fifth night of data collection. The child participants received a copy of the storybook, “Good Night, Dora!”, a box of crayons and a coloring book that was similar, in color and size, to the sleep diary the mother was using. The use of the coloring book was used to incorporate into the study the cultural scripts of familism and collectivism ${ }^{[34,35]}$. These two cultural scripts encourage Latino families include other family members in their day-to-day activities. In addition, the use of the coloring book facilitated the inclusion of the child as an active participant in the study. As compensation for their time, mothers were given a cash card in the value of $\$ 75$ for participating in the study.

\subsection{Measurements}

\subsubsection{Actigraphy}

Maternal and child rest/activity patterns were recorded continuously over five consecutive 24-hour days using a battery-operated actigraphy monitor (Actiwatch, Mini Mitter, Sun River, OR). The Actiwatch detects motion using an accelerometer and translates the recordings into activity counts per epoch. The activity count provides a record of rest and activity that can be scored automatically by a computer algorithm (Actiware, Mini Mitter, Sun River, OR). The actigraphy monitors were set to record at one-minute epochs and zero crossing mode. Actigraphy has been shown to be a reliable and 
valid method to record rest/activity patterns in preschool children ${ }^{[36]}$ and adults ${ }^{[37]}$. In order to obtain reliable measures of sleep patterns for the children in this study, sleep data was collected from the mother and child for 5 days ${ }^{[36]}$.

\subsubsection{Maternal/ child sleep diary}

The mothers recorded their preschool child's and their own sleep patterns for 5 days. The sleep diary assessed bedtime (the time when the mother and child went to bed at night) and rise time (the time that the mother and child got out of bed in the morning). The sleep diary asked questions on the intake of beverages and foods that contained caffeine, medications, bedtime behavior from the previous night, and other events that could affect their or their child's sleep. The sleep diary also incorporated a 24-hour sleep log, which was divided into 30-minute epochs where the mothers documented bedtime and time out of bed. Maternal reports of child rest and activity have shown to be accurate in reporting events like sleep onset and duration ${ }^{[38]}$. The Maternal/Child Sleep Diary and the Maternal Acceptability Survey were translated into Spanish by the first author, back translated by a bilingual nurse scientist, and reviewed by the first author.

\subsubsection{Maternal acceptability survey}

To assess maternal acceptability, a 7-item survey was administered at the end of the data collection. The survey rated maternal acceptability and burden of participating in the study on a 5-point Likert scale. Participants rated keeping track of sleep and wake times in the sleep diary, wearing comfort of the actigraphy monitor, the monitor's degree of interference with daily activity, their willingness to wear the monitor again, and the likelihood that they would recommend the study to other mothers. Two open-ended questions were asked: (1) What were the difficulties that you had in this study? and (2) What suggestion do you have to improve this study?

\subsubsection{Demographic sheet}

An investigator-designed demographic sheet was used to collect demographic information. The demographic data collected was maternal and preschooler age and maternal employment, marital status, employment, and education.

\subsection{Data analysis}

Maternal adherence to actigraphy and sleep diaries was summarized by percentage of missing data from the actigraph and completed sleep diaries. Actigraphy data not recorded for more than 30 minutes and not documented in the sleep diary was assumed to be missing data. Frequencies were used to summarize the mothers' responses on the Maternal Acceptability Survey. Answers to open-ended questions were evaluated for similarities and summarized.

\section{Results}

\subsection{Sample characteristics}

Ten mother-child dyads were recruited and consented to participate in the study during the spring of 2007. Table 1 summarized participant demographics characteristics. All 10 dyads participated in the 5 nights/days of data collection. Maternal night time sleep duration was $7.04 \mathrm{hrs}(\mathrm{SD}+0.84)$. Child night time sleep duration was $7.38 \mathrm{hrs}(\mathrm{SD}+1.32)$.

\subsection{Adherence to data collection protocol}

Adherence to using the actigraph was as follows: One mother-child dyad had periods of missing actigraphy data during daytime hours over the last 3 days of data collection (15\% of their total recording time) but did not have missing actigraphy data for the 5 nights. In looking at the participant's answers to the Maternal Acceptability Survey, the participant did comment that she feared that her child would break the actigraph if she was not watching him. In looking at the actigraph data, the days when actigraph data was missing, for both the mother and the child, were days in which the child was attending daycare. One child had missing actigraph data for 3 continuous day/nights. The missing data could be due to an equipment malfunction or because the child did not wear the actigraph. The Maternal Acceptability Survey from this 
participant did not indicate any difficulties encountered during the study. Therefore, it is unclear why the data was missing from this participant.

Table 1. Demographic characteristics

\begin{tabular}{ll}
\hline Characteristic & Mean (SD) or Frequency \\
\hline Mom age & $27.50(4.6)$ \\
Years in US & $7.10(5.4)$ \\
Children ages & 3 \\
2 years & 2 \\
3 years & 3 \\
4 years & 2 \\
5 years & \\
Marital Status & 9 \\
$\quad$ Married & 1 \\
$\quad$ Living with partner & \\
Employment & 8 \\
$\quad$ Fulltime & 2 \\
$\quad$ Not employed & \\
Years of education & \\
In Mexico & \\
$\quad<6^{\text {th }}$ grade & 3 \\
$\quad 2^{\text {th }}$ grade & 3 \\
$\quad>12^{\text {th }}$ grade & 3 \\
In the U.S. & \\
$7^{\text {th }}-12^{\text {th }}$ grade & 1 \\
Language & \\
$\quad$ Spanish & 9 \\
Bilingual & 1 \\
\hline
\end{tabular}
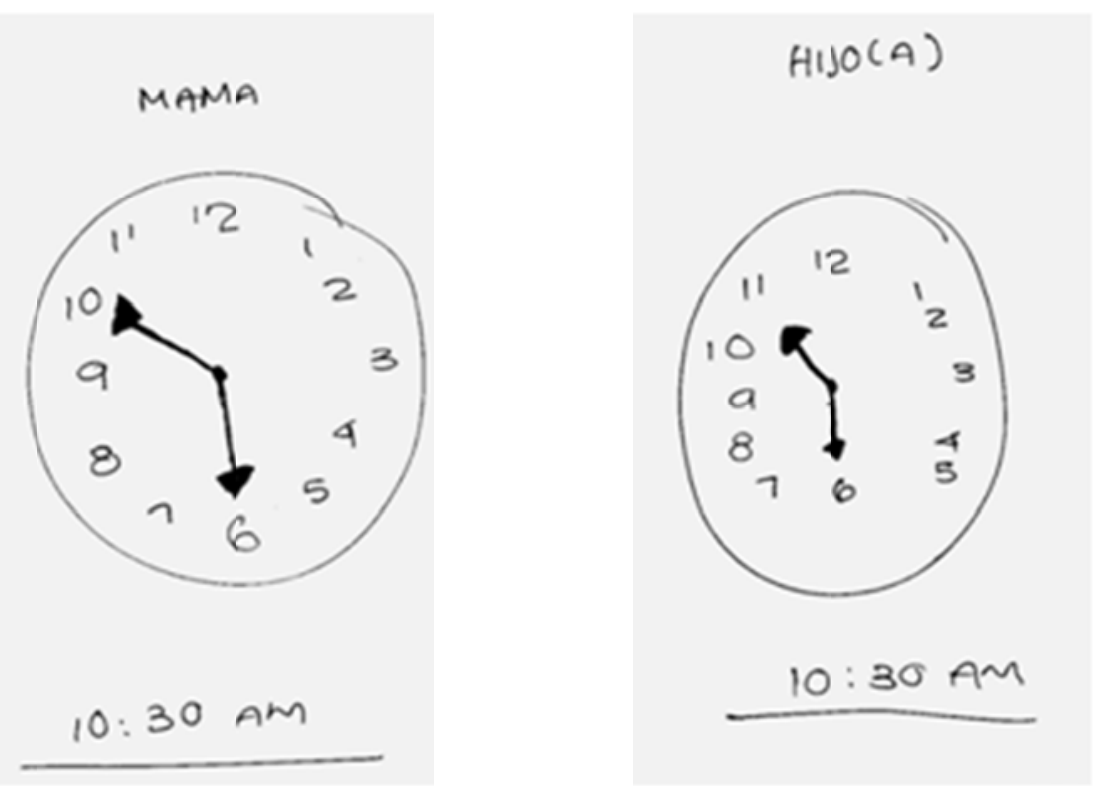

Figure 3. Example of participant's idea of using a clock to replace the sleep log in the sleep diary 
The adherence to the sleep diary had mixed results. There was a 100\% completion rate for the questions on the sleep diary. However, the completion percentage for the 24-hour sleep log in the sleep diary was different. Four mothers had incomplete 24-hour sleep log data for all 5 recording days, and two mothers had incomplete 24-hour sleep log data for the last recording day. When looking at the responses on the Maternal Acceptability Survey, none of these participants mentioned difficulties with the sleep diary. However, as answer to one of the open ended questions in the survey, two participants did suggest that the log should be replaced by a drawing of a clock (See Figure 3).

\subsection{Maternal acceptability to data collection protocol}

The results of the Maternal Acceptability Survey can be seen in Table 2. Nine (90\%) of the maternal participants agreed that it was easy to keep track of their children's and their own rest/activity using the sleep diary. Eight (80\%) of the mothers agreed that wearing the actigraph was comfortable. Nine (90\%) of the mothers agreed that the actigraph did not interfere with their activity, they would be willing to wear the actigraph again, and it was easy to record their child's and their bedtime and rise time using the sleep diary. All (100\%) of the participants would recommend other mothers participate in the study.

From the first open-ended question, 7 out of the 10 participants described the difficulties they experienced while participating in the study. Two participants voiced concerns about the actigraph when they were not with the child. When asked for clarification, several participants explained that they did not want to be financially responsible if their child damaged or lost the actigraph. Two other mothers described difficulties related to the personal discipline required to participate in the study. These difficulties were filling out the forms and having to remove the actigraph before taking a bath. One participant stated that she had difficulty becoming accustomed to wearing the actigraph the first night. One participant stated that the actigraph band bothered her child initially. Lastly, one participant stated that she was not sure if the actigraph event marker was working correctly when she pressed it.

Table 2. Results of Likert scale items on maternal acceptability survey

\begin{tabular}{|c|c|}
\hline Question & Participant Response Frequency \\
\hline Keeping track of my and my child's activity using the diary was: & $\begin{array}{l}\text { 1-Neutral } \\
\text { 7-Easy } \\
\text { 2-Very easy }\end{array}$ \\
\hline Wearing the activity monitor was: & $\begin{array}{l}\text { 1-Some discomfort experienced } \\
\text { 1-Neutral } \\
\text { 4-Comfortable } \\
\text { 4-Very comfortable }\end{array}$ \\
\hline The monitor interfered with my activity: & $\begin{array}{l}\text { 1-It interfered minimally with my activity } \\
\text { 9-It did not interfere with my activity }\end{array}$ \\
\hline I would be willing to wear the monitor again: & $\begin{array}{l}\text { 1-Maybe } \\
\text { 3-Yes } \\
\text { 6-Definitely }\end{array}$ \\
\hline I would recommend other mothers to participate in this study: & $\begin{array}{l}\text { 5-Yes } \\
\text { 5-Definitely }\end{array}$ \\
\hline
\end{tabular}

From the second open-ended question, which asked the participants to provide information on how to improve the study, 5 mothers did not provide suggestions. Two participants stated that the study should go for a longer period of time. When asked why, they explained that they believed it would provide a clearer picture of what sleep related behaviors were like in a household. Three mothers suggested that the 24-hour sleep log should be changed because it was confusing to them. Out of these three mothers, two suggested that the 24-hour log in the sleep diary should be replaced by the picture of a clock. One participant provided a drawing to illustrate her ideas related to the use of a clock (see Figure 3). 


\section{Discussion}

The purpose of this study was to evaluate the feasibility of using actigraphy and sleep diaries with Latino mothers in a community setting by assessing the maternal adherence and acceptance to the data collection methods. Several of the mothers in this study adhered to and accepted the use of actigraphy and sleep diaries as methods to assess sleep in the community setting. However, some of the participants did experience difficulties related to the data collection methods used. There was a mismatch between the answers obtained in the survey questions and the open-ended questions in the Maternal Acceptability Survey. Based on the survey questions, it could be assumed that most of the participants did not experience burden while participating in the study. On the other hand, the results from the open-ended questions provided a different perspective of their experience with the study.

A few of the mothers voiced worries about the safekeeping and financial responsibility of the study equipment. This concern of their child damaging the equipment was especially true if the mothers characterized their children as restless or curios $^{[39]}$. To relieve the participants from such concerns in future studies, they will be informed that they will not be held financially responsible for lost or damaged equipment. This information will be discussed and included in the written consent materials. Providing a clear message that explains who is responsible for damaged or misplaced equipment is important for researchers wishing to incorporate biobehavioral measures that require the use of equipment in ethnic minority or low-income communities. If this message is not clearly provided, community members may not wish to participate due to the fear of being financially responsible for damaged or lost equipment.

All of the participants answered the sleep habit question on the sleep diary. However, several of the participants did not complete the 24-hour sleep log. Sleep researchers use the data collected in the 24-hour sleep logs as a way of validating the data the actigraph data collected. The data collected on the 24-hour sleep logs is plotted on a linear frame of reference that parallels the horizontal line of the actigraphy recording (See Figure 4). Frames of reference are mental images that influence how individual interprets visual images they see ${ }^{[40]}$. Frames of reference vary across languages, cultures, and levels of education. For community members who come from cultural or socioeconomic backgrounds that are different from a researcher's background, the frame of reference for a 24-hour time period may be a clock, not a 24-hour log on a horizontal axis. Similar to the issues encountered when collecting data with instruments that use Likert scales ${ }^{[41]}$, sensitivity to community members' frames of reference needs to take place when developing data collection tools to ensure that adequate and accurate data collection takes place.

\section{Day-time: \\ $\begin{array}{lllllllllllllllllllllllllllllllll}7 \mathrm{~A} & 7: 30 & \mathrm{BA} & 8: 30 & 9 \mathrm{~A} & 9: 30 & 10 \mathrm{~A} & 10: 30 & 11 \mathrm{~A} & 11: 30 & 12 \mathrm{P} & 12: 30 & \text { IP } & 1: 30 & 2 \mathrm{P} & 2: 30 & 3 \mathrm{P} & 3: 30 & 4 \mathrm{P} & 4: 30 & 5 \mathrm{P} & 5: 30 & 6 \mathrm{P} & 6: 30 & 7 \mathrm{P}\end{array}$

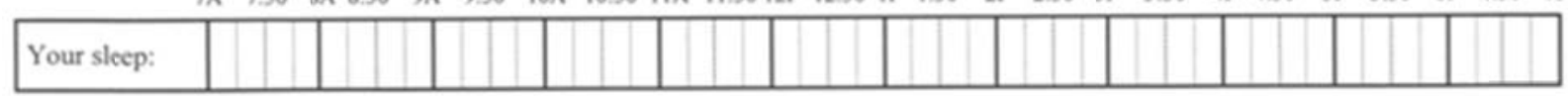

\section{Night-time:}

$\begin{array}{lllllllllllllllllllllllllllllll}7 \mathrm{P} & 7: 30 & 8 \mathrm{P} & 8: 30 & 9 \mathrm{P} & 9: 30 & 10 \mathrm{P} & 10: 30 & \text { IIP } & 11: 30 & 12 \mathrm{~A} & 12: 30 & 1 \mathrm{~A} & 1: 30 & 2 \mathrm{~A} & 2: 30 & 3 \mathrm{~A} & 3: 30 & 4 \mathrm{~A} & 4: 30 & 5 \mathrm{~A} & 5: 30 & 6 \mathrm{~A} & 6: 30 & 7 \mathrm{~A}\end{array}$

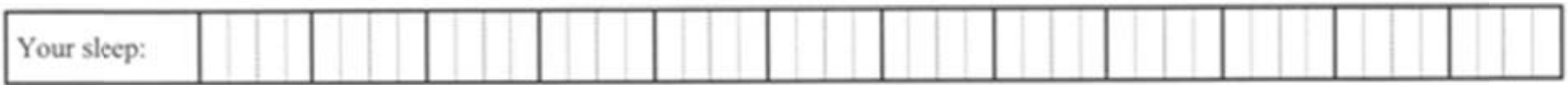

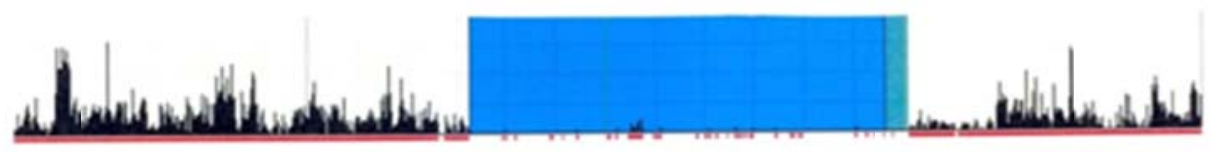

Figure 4. Example of 24-hour sleep log and how it parallels actigraph output 
Some participants also voiced concerns related to the personal discipline needed to follow the study protocol. Specifically, mothers voiced challenges with scheduling time to complete the sleep diary and press the event marker. Although all the participants were provided with a phone number to call if they had any questions or concerns related to the study, none of the participants called. Therefore, in future studies, a reminder call or text message will be offered to participants who wish to be reminded that they need to fill out the sleep diary and press the event marker. Another way of addressing the concerns related to the personal discipline needed to follow the study protocol is to further incorporate the data collection into the participants' daily lives. First, the recording sleep/activity times could be individualized to the participants' daily patterns. The linking of the recording sleep/activity times on the sleep diary with their activities in their lives may ease participant burden. Secondly, the sleep log could be placed on the refrigerator with a magnet. This would serve as a reminder to record sleep/activity data. Lastly, other technologies such as personal digital assistants in cellular phones could be set up with reminders for the participants.

An interesting incident in the study was the children's preference of the actigraph placement. In studies where the sleep was assessed in preschool children ${ }^{[32,33]}$, the actigraphs were placed on the ankle to avoid the child noticing and handling the actigraph, and to prevent the child from accidentally hurting himself or herself with the device. However, in this study where sleep was assessed concurrently in mother and child, the children wanted to emulate their mothers by wearing the actigraph on the wrist. Allowing the child to imitate the mother facilitated the child's cooperation with the adherence of wearing the actigraph. One drawback of placing the actigraphs on children's wrists was that the event marker was pushed multiple times by the children in a few actigraphs, which meant that the actigraph output had several event marks. This resulted in having to use the sleep diary to compare the bedtime and wake time. Therefore, the data obtained from the sleep diary is an important component when performing a study such as the one presented in this manuscript. For this reason, it is crucial that the mothers can use the sleep diary with ease.

This study has limitations. First, all of the participants were born in Mexico. Therefore, the findings can only be applied to women from similar sociodemographic backgrounds. Another limitation was that the Maternal Acceptability Survey assessed the sleep diary as a whole and not the individual sections (i.e. the diary and 24-hour log). One way of correcting this limitation would be to ask a separate question related to completing the sleep diary questions and the sleep log. Lastly, sleep was only assessed during the weekdays. Therefore, it is not known if adherence to actigraphy and sleep diaries would be different during the weekend when sleep schedules typically change are less regular. Despite this limitation, the information that this feasibility study will inform future research studies that wish to use actigraphy and sleep diaries in a community setting.

Based on participant feedback, future data collection protocols will be revised to minimize the burden that some of the participants experienced during this study. The findings in this feasibility study have implications for future nursing biobehavioral studies in the community setting. First, respondent burden must be assessed because it can affect the quality and accuracy of the data collected. Second, investigators must examine their data collection methods to ensure that participants can answer the questions or provide the data requested. Assessing the appropriateness of data collection methods is important with all populations, but it is especially important when working with ethnic or low-income communities. The members of such communities may have different frames of reference than the investigators conducting the study. Lastly, it is important for researchers to address concerns that participants may have related to the study. Potential participant burden, issues with data collection methods, and possible participant concerns can be easily assessed by developing a short survey or using the two open-ended question used in this study: (1) What were the difficulties that you had in this study? and (2) What suggestion do you have to improve this study? Keeping these three implications in mind will facilitate the process of translational research by improving the collection of biobehavioral measures in the community.

\section{Acknowledgements}

This study was funded by the Elizabeth Giblin Research Fund in Nursing from the University of Washington School of Nursing and NIH/NINR T32 NR07039. We would also like to thank Drs. Kathryn Lee from University of California-San 
Francisco and Carol Landis from the University of Washington for their guidance in the methods for study, and the Family Community Center in North Seattle and it member for the opportunity to conduct this study.

\section{References}

[1] Ennis SR, Ríos-Vargas M, Albert NG. The hispanic population: 2010 US Department of Commerce, Economics and Statistics Administration, US Census Bureau. 2011.

[2] Ogden CL, Carroll MD, Kit BK, Flegal KM. Prevalence of obesity and trends in body mass index among US children and adolescents, 1999-2010. JAMA: The Journal of the American Medical Association. 2012; 307(5): 483-490. PMid:22253364 http://dx.doi.org/10.1001/jama.2012.40

[3] Flegal KM, Carroll MD, Kit BK, Ogden CL. Prevalence of obesity and trends in the distribution of body mass index among US adults, 1999-2010. JAMA: The Journal of the American Medical Association. 2012; 307(5): 491-497. PMid:22253363 http://dx.doi.org/10.1001/jama.2012.39

[4] Dietz WH. The obesity epidemic in young children: Reduce television viewing and promote playing. BMJ: British Medical Journal. 2001; 322(7282): 313. PMCid:1119564 http://dx.doi.org/10.1136/bmj.322.7282.313

[5] Skidmore P, Yarnell J. The obesity epidemic: Prospects for prevention. QJM. 2004; 97(12): 817-825. PMid:15569814 http://dx.doi.org/10.1093/qjmed/hch136

[6] Cappuccio FP, Taggart FM, Kandala N, Currie A. Meta-analysis of short sleep duration and obesity in children and adults. Sleep. 2008; 31(5): 619. PMid:18517032

[7] Patel SR, Hu FB. Short sleep duration and weight gain: A systematic review. Obesity. 2012; 16(3): 643-653. PMid:18239586 http://dx.doi.org/10.1038/oby.2007.118

[8] Chen X, Beydoun MA, Wang Y. Is sleep duration associated with childhood obesity? A systematic review and meta-analysis. Obesity. 2012; 16(2): 265-274. PMid:18239632 http://dx.doi.org/10.1038/oby.2007.63

[9] Guidolin M, Gradisar M. Is shortened sleep duration a risk factor for overweight and obesity during adolescence? A review of the empirical literature. Sleep Medicine. 2012. PMid:22633283 http://dx.doi.org/10.1016/j.sleep.2012.03.016

[10] Buxton OM, Pavlova M, Reid EW, Wang W, Simonson DC, Adler GK. Sleep restriction for 1 week reduces insulin sensitivity in healthy men. Diabetes. 2010; 59(9): 2126-2133. PMid:20585000 http://dx.doi.org/10.2337/db09-0699

[11] Javaheri S, Storfer-Isser A, Rosen CL, Redline S. Association of short and long sleep durations with insulin sensitivity in adolescents. The Journal of Pediatrics. 2011; 158(4): 617-623. PMid:21146189 http://dx.doi.org/10.1016/j.jpeds.2010.09.080

[12] Spiegel K, Leproult R, Van Cauter E. Impact of sleep debt on metabolic and endocrine function. The Lancet, 1999; 354(9188): 1435-1439. http://dx.doi.org/10.1016/S0140-6736(99)01376-8

[13] Morselli L, Leproult R, Balbo M, Spiegel K. Role of sleep duration in the regulation of glucose metabolism and appetite. Best Practice \& Research Clinical Endocrinology \& Metabolism. 2010; 24(5): 687-702. PMid:21112019 http://dx.doi.org/10.1016/j.beem.2010.07.005

[14] St-Onge M, O'Keeffe M, Roberts A, Roychoudhury A, Laferrere B. Short sleep duration, glucose dysregulation and hormonal regulation of appetite in men and women. Sleep. 2012; 35(11): 1503-1510. PMid:23115399

[15] Nedeltcheva AV, Kessler L, Imperial J, Penev PD. Exposure to recurrent sleep restriction in the setting of high caloric intake and physical inactivity results in increased insulin resistance and reduced glucose tolerance. Journal of Clinical Endocrinology \& Metabolism. 2009; 94(9): 3242-3250. PMid:19567526 http://dx.doi.org/10.1210/jc.2009-0483

[16] Nedeltcheva AV, Kilkus JM, Imperial J, Kasza K, Schoeller DA, Penev PD. Sleep curtailment is accompanied by increased intake of calories from snacks. American Journal of Clinical Nutrition. 2009; 89(1): 126-133. http://dx.doi.org/10.3945/ajcn.2008.26574

[17] Nishiura C, Noguchi J, Hashimoto H. Dietary patterns only partially explain the effect of short sleep duration on the incidence of obesity. Sleep. 2010; 33(6): 753. PMid:20550015

[18] Theorell-Haglöw J, Lindberg E, Janson C. What are the im-portant risk factors for daytime sleepiness and fatigue in women. Sleep. 2006; 29(6): 751-757. PMid:16796213

[19] Kapsimalis F, Basta M, Varouchakis G, Gourgoulianis K, Vgontzas A, Kryger M. Cytokines and pathological sleep. Sleep Medicine. 2008; 9(6): 603-614. PMid:18024171 http://dx.doi.org/10.1016/j.sleep.2007.08.019

[20] Vgontzas, AN, Bixler, EO, Chrousou, GP. Obesity-Related sleepiness and fatigue. Annals of the New York Academy of Sciences. 2006; 1083(1): 329-344. PMid:17148748 http://dx.doi.org/10.1196/annals.1367.023

[21] Schmid SM, Hallschmid M, Jauch-Chara K, etal. Short-term sleep loss decreases physical activity under free-living conditions but does not increase food intake under time-deprived laboratory conditions in healthy men. The American Journal of Clinical Nutrition. 2009; 90(6): 1476-1482. PMid:19846546 http://dx.doi.org/10.3945/ajcn.2009.27984 
[22] Stone MR, Stevens D, Faulkner GEJ. Maintaining recommended sleep throughout the week is associated with increased physical activity in children. Preventive Medicine. 2013; 56(2): 112-117. http://dx.doi.org/10.1016/j.ypmed.2012.11.015

[23] Clark L. Maternal responsibility for health in the household. Health Care for Women International. 1995; 16(1): 43-55. PMid:7706140 http://dx.doi.org/10.1080/07399339509516156

[24] Mendelson C. Health perceptions of mexican american women. Journal of Transcultural Nursing. 2002; 13(3): $210-217$. PMid:12113152 http://dx.doi.org/10.1177/10459602013003010

[25] Sadeh A, Acebo C. The role of actigraphy in sleep medicine. Sleep Medicine Reviews. 2002; 6(2): 113-124. http://dx.doi.org/10.1053/smrv.2001.0182

[26] Tryon WW. Issues of validity in actigraphic sleep assessment. Sleep. 2004; 27(1): 158-165.

[27] Ustinov Y, Lichstein KL. Actigraphy reliability with normal sleepers. Behavioral Sleep Medicine, (just-accepted). 2012.

[28] Arnold KR. Anti-immigration in the united states: A historical encyclopedia Abc-clio.2011.

[29] Burke NJ, Bird JA, Clark MA, Rakowski W, Guerra C, Barker JC, Pasick RJ. Social and cultural meanings of self-efficacy. Health Education \& Behavior. 2009; 36(5 suppl): 111S-128S.

[30] Caldwell BA, Redeker NS. Sleep patterns and psychological distress in women living in an inner city. Research in Nursing \& Health. 2009; 32(2): 177-190. PMid:19086035 http://dx.doi.org/10.1002/nur.20308

[31] Ulrich CM, Wallen GR, Feister A, Grady C. Respondent burden in clinical research: When are we asking too much of subjects? IRB: Ethics and Human Research. 2005; 27(4): 17-20. PMid:16220630 http://dx.doi.org/10.2307/3563957

[32] Acebo C, Sadeh A, Seifer R, Tzischinsky O, Hafer A, Carskadon MA. Sleep/wake patterns derived from activity monitoring and maternal report for healthy 1-to 5-year-old children. Sleep. 2005; 28(12): 1568. PMid:19805794 http://dx.doi.org/10.1177/1090198109338916

[33] Ward TM, Gay C, Alkon A, Anders TF, Lee KA. Nocturnal sleep and daytime nap behaviors in relation to salivary cortisol levels and temperament in preschool-age children attending child care. Biological Research for Nursing. 2008; 9(3): 244-253. PMid:18077777 http://dx.doi.org/10.1177/1099800407310158

[34] Marin G, Marin BV. Research with hispanic populations. Sage Publications, Inc. 1991.

[35] Padilla YC, Villalobos G. Cultural responses to health among mexican american women and their families. Family \& Community Health. 2007; 30: S24-S33. PMid:17159629 http://dx.doi.org/10.1097/00003727-200701001-00005

[36] Acebo C, Sadeh A, Seifer R, Tzischinsky O, Wolfson A, Hafer A, Carskadon M. Estimating sleep patterns with activity monitoring in children and adolescents: How many nights are necessary for reliable measures? Sleep.1999; 22(1): 95. PMid:9989370

[37] Littner, MM, Kushida CA, Dennis Bailey D, Berry RB, Davila DG, Hirshkowitz M. Practice parameters for the role of actigraphy in the study of sleep and circadian rhythms: An update for 2002. Sleep. 2003; 26(3): 337. PMid:12749556

[38] Werner H, Molinari L, Guyer C, Jenni OG. Agreement rates between actigraphy, diary, and questionnaire for children's sleep patterns. Archives of pediatrics \& adolescent medicine. 2008; 162(4): 350.

[39] Gallagher MR, Reifsnider E, Gill S. Home-Based injury prevention practices used by mothers of mexican descent living in san antonio, TX. Public Health Nursing. 2009; 26(2): 114-123. PMid:19261150 http://dx.doi.org/10.1111/j.1525-1446.2009.00762.x

[40] Majid A, Bowerman M, Kita S, Haun D, Levinson SC. Can language restructure cognition? the case for space. Trends in Cognitive Sciences. 2004; 8(3): 108-114. PMid:15301750 http://dx.doi.org/10.1016/j.tics.2004.01.003

[41] Flaskerud JH. Is the Likert Scale Format Culturally Biased? Nursing Research. 1988; 37(3): 185-186. PMid:3368361 http://dx.doi.org/10.1097/00006199-198805000-00013 